ESAIM: PROCEEDINGS, Juillet 2013, Vol. 40, p. 63-82

C. Bourdarias, S. Gerbi, Editors

\title{
APPROXIMATE SOLUTIONS OF THE BAER-NUNZIATO MODEL
}

\author{
Fabien Crouzet, Frédéric Daude ${ }^{1}$, Pascal Galon ${ }^{2}$, Philippe Helluy ${ }^{3}$, \\ Jean-Marc Hérard, Olivier Hurisse ${ }^{4}$ and Yujie Liu ${ }^{5}$
}

\begin{abstract}
We examine in this paper the accuracy of some approximations of the BaerNunziato two-phase flow model. The governing equations and their main properties are recalled, and two distinct numerical schemes are investigated, including a classical secondorder extension relying on symmetrizing variables. Shock tube cases are considered, and two simple Riemann problems based on well-balanced initial data are detailed. These enable to recover the expected convergence rates. However, it is shown that these simple cases are indeed very difficult and that the accuracy of basic schemes is rather poor.

Résumé. Approximation des solutions du modèle de Baer-Nunziato On examine ici la précision des approximations obtenues pour le modèle diphasique de BaerNunziato. Les équations du modèle et ses principales propriétés sont rappellées. Deux schémas distincts sont proposés, et des extensions classiques au second-ordre sont considérées, utilisant les variables de symétrisation. Des cas tests de tube à choc sont analysés, notamment deux cas utilisant des conditions initiales en équilibre. Les taux de convergence attendus sont retrouvés, mais on montre que la précision des approximations de certains problèmes de Riemann est assez médiocre.
\end{abstract}

\section{INTRODUCTION}

Several two-phase flow models may be used in order to predict water-hammer phenomena in pipes. Though the homogeneous approach is widely used for that purpose, the two-fluid model is also a rather appealing approach. Among these models, the Baer-Nunziato model $[4,6]$ arises at once, especially in this framework involving fast transient flows together with strong rarefactions and compressible effects. Unlike some other candidates, its hyperbolic structure and unique jump

\footnotetext{
${ }^{1}$ EDF, R\&D, AMA, and LaMSID, UMR EDF/CNRS/CEA 2832, 1 avenue du Général de Gaulle, 92141, Clamart, France

2 CEA, Saclay, France, and LaMSID, UMR EDF/CNRS/CEA 2832, 1 avenue du Général de Gaulle, 92141, Clamart, France

${ }^{3}$ IRMA, 7 rue Descartes, Université de Strasbourg, 67084, Strasbourg, France

${ }^{4} \mathrm{EDF}, \mathrm{R} \& \mathrm{D}$, MFEE, 6 quai Watier, 78400, Chatou, France

5 EDF, R\&D, AMA, and LaMSID, UMR EDF/CNRS/CEA 2832, 1 avenue du Général de Gaulle, 92141, Clamart, France. PhD student in LATP-Université Aix-Marseille, 39 rue Joliot Curie, 13453, Marseille, France
}

(C) EDP Sciences, SMAI 2013 
conditions render the computation of approximate solutions meaningful, at least from a mathematical point of view. The difficult question of whether it enables to obtain accurate enough predictions is precisely one main goal for the SITAR project.

Nonetheless, this rather complex model contains many non-linear waves, and even more two distinct contact discontinuities, which are known to slow down the convergence towards exact solutions. One contact discontinuity is connected with the velocity of the dilute vapour phase, whereas the second one corresponds to the velocity of the liquid phase. Thus, we expect to observe a rather poor accuracy on coarse meshes, due to the fact that none among existing schemes preserves the so-called statistical fraction waves (which will be defined in the sequel). The fact that the two linearly degenerated (LD) fields are close to one another renders the problem even more tricky. It is well known from a long time that the excellent Godunov scheme may provide rather poor approximations of solutions of single-phase Euler equations on coarse meshes, even when the equations of state are not too intricate (stiffened gas EOS or Mie-Gruneisen EOS for instance). Several recipes have been proposed in the literature since the early papers by Karni and Abgrall [1], which means that the amplitude of oscillations around the contact discontinuity may be considerably reduced, using suitable modifications of algorithms. However, as emphasized in [11], approximate solutions may converge towards wrong shock solutions when present in the field, due to the fact that non-conservative schemes are widely used to smooth profiles. This in turn means that classical conservative Finite Volume schemes cannot be disregarded.

Hence, one main objective in this paper consists in verifying the convergence of approximate solutions of the Baer-Nunziato model towards the correct solutions when the mesh is refined, and also in evaluating the accuracy of some simple and cheap schemes on rather coarse meshes. This implies that well-balanced initial data need to be defined and used in order to initialize one-dimensional Riemann problems for the Baer-Nunziato model. This will be detailed, once the governing equations, the main properties, and Finite Volume schemes have been recalled and/or defined. Modelling issues and numerical problems arising with the full set of equations including interfacial source terms will not be discussed herein, and the reader is refered to $[2,3,7,10,12-17,19,21,22]$ among others.

\section{Governing equations of the Baer-Nunziato model}

Throughout the paper, indexes $l, v$ will refer to the liquid and vapour phases. The statistical void fractions of the vapour phase and the liquid phase are noted $\alpha_{v}$ and $\alpha_{l}$ respectively, and the local constraint $\alpha_{l}=1-\alpha_{v}$ holds everywhere. We classically note liquid and vapour pressures $P_{l, v}$, phasic velocities $U_{l, v}$ and densities $\rho_{l, v}$. Partial masses are noted $m_{\phi}=\alpha_{\phi} \rho_{\phi}$ for $\phi=v, l$. Equations of state are given by the user, hence functions $e_{\phi}\left(P_{\phi}, \rho_{\phi}\right)$ are known explicitly, so that the total energy is:

$$
E_{\phi}=\rho_{\phi} e_{\phi}\left(P_{\phi}, \rho_{\phi}\right)+\rho_{\phi} \frac{U_{\phi}^{2}}{2}, \quad \phi=v, l
$$

The state variable $W$ of the Baer-Nunziato model is defined by:

$$
W=\left(\alpha_{l}, \alpha_{l} \rho_{l}, \alpha_{l} \rho_{l} U_{l}, \alpha_{l} E_{l}, \alpha_{v} \rho_{v}, \alpha_{v} \rho_{v} U_{v}, \alpha_{v} E_{v}\right)
$$


We focus in the paper on flows dominated by the liquid phase, and thus assume that the statistical fraction $\alpha_{v}$ is small compared with 1 .

When neglecting source terms associated with drag effects, but also interfacial heat and mass transfer, the governing set of equations of the Baer-Nunziato model may be written as follows $[4,6]$ :

$$
\left\{\begin{array}{l}
\partial_{t}\left(\alpha_{l}\right)+U_{v} \partial_{x}\left(\alpha_{l}\right)=0 \\
\partial_{t}\left(\alpha_{l} \rho_{l}\right)+\partial_{x}\left(\alpha_{l} \rho_{l} U_{l}\right)=0 \\
\partial_{t}\left(\alpha_{l} \rho_{l} U_{l}\right)+\partial_{x}\left(\alpha_{l} \rho_{l} U_{l}^{2}+\alpha_{l} P_{l}\right)-P_{l} \partial_{x}\left(\alpha_{l}\right)=0 \\
\partial_{t}\left(\alpha_{l} E_{l}\right)+\partial_{x}\left(\alpha_{l} U_{l}\left(E_{l}+P_{l}\right)\right)+P_{l} \partial_{t}\left(\alpha_{l}\right)=0 \\
\partial_{t}\left(\alpha_{v} \rho_{v}\right)+\partial_{x}\left(\alpha_{v} \rho_{v} U_{v}\right)=0 \\
\partial_{t}\left(\alpha_{v} \rho_{v} U_{v}\right)+\partial_{x}\left(\alpha_{v} \rho_{v} U_{v}^{2}+\alpha_{v} P_{v}\right)-P_{l} \partial_{x}\left(\alpha_{v}\right)=0 \\
\partial_{t}\left(\alpha_{v} E_{v}\right)+\partial_{x}\left(\alpha_{v} U_{v}\left(E_{v}+P_{v}\right)\right)+P_{l} \partial_{t}\left(\alpha_{v}\right)=0
\end{array}\right.
$$

Source terms may be incorporated using the fractional step method, when these comply with the overall entropy inequality. Some algorithms have been proposed in $[10,17]$ for that purpose. The celerity of acoustic waves in the pure liquid (respectively vapour) phase is noted $c_{l}$ (resp. $c_{v}$ ), and the phase entropy $s_{\phi}$ complies with:

$$
\left(c_{\phi}\right)^{2} \partial_{P_{\phi}}\left(s_{\phi}\right)+\partial_{\rho_{\phi}}\left(s_{\phi}\right)=0
$$

We recall below the main properties of system (2) (details can be found in $[3,12]$ ).

\section{Property 1}

- Hyperbolicity:

The set of equations (2) is hyperbolic. It admits seven real eigenvalues:

$\lambda_{1,2}=U_{v}, \quad \lambda_{3}=U_{v}-c_{v}, \quad \lambda_{4}=U_{v}+c_{v}, \quad \lambda_{5}=U_{l}, \quad \lambda_{6}=U_{l}-c_{l}, \quad \lambda_{7}=U_{l}+c_{l}$

and associated right eigenvectors span the whole space $\mathcal{R}^{7}$, unless $\left|U_{l}-U_{v}\right| / c_{l}=1$.

- Entropy inequality:

Define the entropy $\eta(W)=m_{l} s_{l}+m_{v} s_{v}$ and the entropy flux $f_{\eta}(W)=m_{l} s_{l} U_{l}+m_{v} s_{v} U_{v}$; then smooth solutions $W$ of (2) are such that:

$$
0=\partial_{t}(\eta(W))+\partial_{x}\left(f_{\eta}(W)\right) .
$$

When diffusive and source terms are taken into account, the latter equality turns into the following inequality:

$$
0 \leq \partial_{t}(\eta(W))+\partial_{x}\left(f_{\eta}(W)\right)
$$

- Structure of fields:

Fields associated with eigenvalues $\lambda_{1,2,5}$ are linearly degenerate (LD). Other fields are genuinely non linear (GNL). 
- Riemann invariants through LD waves:

The five Riemann invariants of the $1-2$ LD field associated with the void fraction coupling wave are the following:

$$
\begin{aligned}
& I_{1-2}^{1-}(W)=U_{v} \\
& I_{1-2}^{2}(W)=s_{l} ; \\
& I_{1-2}^{3}(W)=m_{l}\left(U_{l}-U_{v}\right) \\
& I_{1-2}^{4}(W)=\alpha_{l} P_{l}+\alpha_{v} P_{v}+m_{l}\left(U_{v}-U_{l}\right)^{2} \\
& I_{1-2}^{5}(W)=e_{l}+P_{l} / \rho_{l}+\left(U_{v}-U_{l}\right)^{2} / 2 .
\end{aligned}
$$

The structure of the $5 \mathrm{LD}$ wave is classical, since:

$$
\begin{aligned}
& I_{5}^{1}(W)=U_{l} \quad ; \quad I_{5}^{2}(W)=P_{l} ; \\
& I_{5}^{3}(W)=\alpha_{l} \quad ; \quad I_{5}^{4}(W)=P_{v} ; \\
& I_{5}^{5}(W)=U_{v} \quad ; \quad I_{5}^{6}(W)=\rho_{v} .
\end{aligned}
$$

- Jump conditions:

Within each isolated field associated with $\lambda_{k}=3,4,6,7$, unique jump conditions hold. If $\sigma$ denotes the speed of the shock wave, and $l, r$ the left-right states on each side of this travelling discontinuity, these jump conditions are:

$$
\begin{aligned}
& {\left[\alpha_{v}\right]_{l}^{r}=0} \\
& -\sigma\left[\rho_{\phi}\right]_{l}^{r}+\left[\rho_{\phi} U_{\phi}\right]_{l}^{r}=0 \\
& -\sigma\left[\rho_{\phi} U_{\phi}\right]_{l}^{r}+\left[\rho_{\phi} U_{\phi}^{2}+P_{\phi}\right]_{l}^{r}=0 \\
& -\sigma\left[E_{\phi}\right]_{l}^{r}+\left[U_{\phi}\left(E_{\phi}+P_{\phi}\right)\right]_{l}^{r}=0
\end{aligned}
$$

and thus shock relations are exactly single-phase shock relations, field by field. Moreover, Riemann invariants of the latter 3,4 waves (and 6, 7 waves respectively) coincide with those of the pure single vapour (respectively liquid) phase.

The structure of Riemann invariants detailed in (6) enables to build basic solutions of the Riemann problem, such as pure statistical fraction waves for instance (see below). The entropy inequality (5) is obtained assuming vanishing viscosity contributions; when focusing on shock waves associated with 3,4,6, 7 fields, it corresponds to the classical entropy inequality for Euler equations, and thus selects the correct shock solution in (8).

\section{NumericAl SCHEMES}

\subsection{First-order and second-order Rusanov scheme}

System (2) may be recast in the following form:

$$
\partial_{t}(W)+\partial_{x}(\mathcal{F}(W))+\mathcal{H}(W) \partial_{x}\left(\alpha_{l}\right)=0
$$

while noting:

$$
\begin{gathered}
\mathcal{F}(W)=\left(0, m_{\phi} U_{\phi}, m_{\phi} U_{\phi}^{2}+\alpha_{\phi} P_{\phi}, \alpha_{\phi} U_{\phi}\left(E_{\phi}+P_{\phi}\right)\right) \\
\mathcal{H}(W)=\left(U_{v}, 0,-P_{l},-P_{l} U_{v}, 0, P_{l}, P_{l} U_{v}\right)
\end{gathered}
$$


The first-order Rusanov scheme (refered to as SR1 in the sequel) computes approximations of solutions of system (9) as follows. If $\Delta t$ denotes the time step and $h_{i}$ is the size of cell $\Omega_{i}$, it computes $W_{i}^{n+1}$ in terms of neighbouring cell values $W_{k}^{n}($ for $k=i-1, i, i+1)$ using a three-point scheme:

$$
h_{i}\left(W_{i}^{n+1}-W_{i}^{n}\right)+\Delta t\left(f_{i+1 / 2}^{\text {Rusanov }}\left(W_{i}^{n}, W_{i+1}^{n}\right)-f_{i-1 / 2}^{\text {Rusanov }}\left(W_{i-1}^{n}, W_{i}^{n}\right)\right)+\Delta t \mathcal{T}_{i}^{n}=0
$$

The numerical flux $f_{i+1 / 2}^{R u s a n o v}\left(W_{i}^{n}, W_{i+1}^{n}\right)$ through the interface $(i+1 / 2)$ separating cells $i$ and $i+1$ is defined by:

$$
f_{i+1 / 2}^{\text {Rusanov }}\left(W_{i}^{n}, W_{i+1}^{n}\right)=\left(\left(\mathcal{F}\left(W_{i}^{n}\right)+\mathcal{F}\left(W_{i+1}^{n}\right)\right)-r_{i+1 / 2}\left(W_{i+1}^{n}-W_{i}^{n}\right)\right) / 2
$$

In this formula, the scalar $r_{i+1 / 2}$ is equal to $\max \left(R_{i}^{n}, R_{i+1}^{n}\right)$ where $R_{k}^{n}$ represents the spectral radius of the whole convection matrix associated with (9) and estimated at $W_{k}^{n}$, for $k=i, i+1$ :

$$
R_{k}^{n}=\max \left(\left|\left(U_{l}\right)_{k}^{n}\right|+\left(c_{l}\right)_{k}^{n},\left|\left(U_{v}\right)_{k}^{n}\right|+\left(c_{v}\right)_{k}^{n}\right)
$$

If $\bar{\phi}_{k+1 / 2}^{n}$ stands for the mean value $\left(\phi_{k}^{n}+\phi_{k+1}^{n}\right) / 2$, the non-conservative contribution $\mathcal{T}_{i}^{n}$ is calculated as:

$$
\mathcal{T}_{i}^{n}=\mathcal{H}\left(W_{i}^{n}\right)\left(\left(\bar{\alpha}_{l}\right)_{i+1 / 2}^{n}-\left(\bar{\alpha}_{l}\right)_{i-1 / 2}^{n}\right)
$$

We recall that the first-order Rusanov scheme enjoys the following property:

\section{Property 2:}

Assuming that the time step complies with the condition:

$$
\Delta t\left(r_{i-1 / 2}+r_{i+1 / 2}\right)<2 h_{i}
$$

then scheme (10), (11), (12) preserves positive values of partial masses $m_{\phi}$ and void fractions $\alpha_{\phi}$, for $\phi=l, v$.

\section{Second order extension of Rusanov scheme}

The second-order Rusanov scheme SR1-ORDER2 relies on a classical minmod reconstruction of the symmetrizing variable $Z^{t}=\left(\alpha_{l}, U_{l}, U_{v}, P_{l}, P_{v}, s_{l}, s_{v}\right)$ within each cell $i$ (see [9]). Hence, at each time step, we define within each cell a linear reconstruction for $Z$ :

$$
Z_{i}\left(x, t^{n}\right)=Z_{i}^{n}+\left(x-x_{i}\right)(\nabla Z)_{i}^{n}
$$

where:

$$
\left.h_{i}(\nabla Z)_{i}^{n}=\operatorname{sign}\left(Z_{i+1}^{n}-Z_{i}^{n}\right) \dot{(m i n}\left(\left|Z_{i+1}^{n}-Z_{i}^{n}\right|,\left|Z_{i-1}^{n}-Z_{i}^{n}\right|\right)\right)
$$

if

and $(\nabla Z)_{i}^{n}=0$ otherwise.

$$
\left(Z_{i+1}^{n}-Z_{i}^{n}\right)\left(Z_{i}^{n}-Z_{i-1}^{n}\right)>0
$$

These enable to define values $W_{i+1 / 2}^{-}=W\left(Z_{i+1 / 2}^{-}\right)$where: $Z_{i+1 / 2}^{-}=Z_{i}^{n}+h_{i}(\nabla Z)_{i}^{n} / 2$ and: $W_{i+1 / 2}^{+}=W\left(Z_{i+1 / 2}^{+}\right)$where: $Z_{i+1 / 2}^{+}=Z_{i+1}^{n}-h_{i+1}(\nabla Z)_{i+1}^{n} / 2$, on both sides on the interface $i+1 / 2$. Thus the counterpart of the first-order numerical flux $f_{i+1 / 2}^{\text {Rusanov }}\left(W_{i}^{n}, W_{i+1}^{n}\right)$ in (10) is now : 


$$
f_{i+1 / 2}^{\text {Rusanov }-2}\left(W_{i-1}^{n}, W_{i}^{n}, W_{i+1}^{n}, W_{i+2}^{n}\right)=\left(\left(\mathcal{F}\left(W_{i+1 / 2}^{-}\right)+\mathcal{F}\left(W_{i+1 / 2}^{+}\right)\right)-r_{i+1 / 2}\left(W_{i+1 / 2}^{+}-W_{i+1 / 2}^{-}\right)\right) / 2
$$

The term $\mathcal{T}_{i}^{n}$ remains exactly the same.

For time integration we use a second-order Runge-Kutta time scheme. This completes the description of first and second-order Rusanov schemes.

\subsection{A fractional step method to compute Baer-Nunziato model}

We present now a second scheme relying on the fractional step method. The fractional step method is very useful in an industrial framework, since it allows to cope with simpler systems, and also provides a rather stable strategy. The fractional step approach has been used many years ago by Baraille, Leroux (see [5]) and other authors in order to obtain approximate solutions of Euler equations on structured and unstructured meshes. The following scheme should not be confused with another fractional step method introduced in [7,20]. Starting with an initial condition $W_{i}^{n}$, we compute first some approximation of solutions of:

Step 1:

$$
\left\{\begin{array}{l}
\partial_{t}\left(\alpha_{l}\right)+U_{v} \partial_{x}\left(\alpha_{l}\right)=0 \\
\partial_{t}\left(\alpha_{\phi} \rho_{\phi}\right)=0 \\
\partial_{t}\left(\alpha_{\phi} \rho_{\phi} U_{\phi}\right)=0 \\
\partial_{t}\left(\alpha_{l} E_{l}+\alpha_{v} E_{v}\right)=0 \\
\partial_{t}\left(\alpha_{l} E_{l}\right)+P_{l} \partial_{t}\left(\alpha_{l}\right)=0
\end{array}\right.
$$

for $\phi=l, v$. Then, using final values $\hat{W}_{j}$ as an initial condition, we compute approximate solutions of:

Step 2:

$$
\left\{\begin{array}{l}
\partial_{t}\left(\alpha_{l}\right)=0 \\
\partial_{t}\left(\alpha_{\phi} \rho_{\phi}\right)+\partial_{x}\left(\alpha_{\phi} \rho_{\phi} U_{\phi}\right)=0 \\
\partial_{t}\left(\alpha_{\phi} \rho_{\phi} U_{\phi}\right)+\partial_{x}\left(\alpha_{\phi} \rho_{\phi} U_{\phi}^{2}+\alpha_{\phi} P_{\phi}\right)-P_{l} \partial_{x}\left(\alpha_{\phi}\right)=0 \\
\partial_{t}\left(\alpha_{\phi} E_{\phi}\right)+\partial_{x}\left(\alpha_{\phi} U_{\phi}\left(E_{\phi}+P_{\phi}\right)\right)=0
\end{array}\right.
$$

The previous fractional step method PFRAC3 enjoys the following properties:

\section{Proposition 1:}

- Structure of step 1:

System (14) is hyperbolic. It admits seven real eigenvalues: $\lambda_{1}=U_{v} \quad \lambda_{2-7}=0$ and associated right eigenvectors span $\mathcal{R}^{7}$. All fields are linearly degenerated.

- Riemann invariants in step 1:

The six Riemann invariants through the 1-field are:

$$
R_{u_{v}}=\left\{m_{l}, m_{v}, U_{l}, U_{v}, s_{l}, m_{l} e_{l}+m_{v} e_{v}\right\}
$$


The sole Riemann invariant of the $2-7$-wave is $\alpha_{l}$.

- Structure of step 2:

System (15) is hyperbolic. It admits seven real eigenvalues:

$$
\begin{aligned}
& \lambda_{1}=0 \\
& \lambda_{2}=U_{v}, \quad \lambda_{3}=U_{v}-c_{v}, \quad \lambda_{4}=U_{v}+c_{v}, \\
& \lambda_{5}=U_{l}, \quad \lambda_{6}=U_{l}-c_{l}, \quad \lambda_{7}=U_{l}+c_{l}
\end{aligned}
$$

and associated right eigenvectors span $\mathcal{R}^{7}$, unless: $\left|U_{l}\right|=c_{l}$, or: $\left|U_{v}\right|=c_{v}$. The 1,2,5-fields are $L D$ and other fields are genuinely non linear.

- Riemann invariants in step 2:

Focusing on step 2, we note that Riemann invariants of the steady 1-wave are:

$$
R_{0}=\left\{m_{l} U_{l}, m_{v} U_{v}, H_{l}, H_{v}, \Sigma_{k=l, v}\left(m_{k} U_{k}^{2}+\alpha_{k} P_{k}\right), s_{l}\right\}
$$

setting $H_{k}=e_{k}+P_{k} / \rho_{k}+U_{k}^{2} / 2$. Moreover, Riemann invariants associated with the other waves are respectively:

$$
\left\{\begin{array}{l}
R_{u_{v}}=\left(\alpha_{v}, u_{v}, p_{v}, u_{l}, p_{l}, s_{l}\right) \\
R_{u_{v} \pm c_{v}}=\left(\alpha_{v}, s_{v}, u_{v} \pm f_{v}, u_{l}, p_{l}, s_{l}\right) \\
R_{u_{l}}=\left(\alpha_{v}, u_{v}, p_{v}, s_{v}, u_{l}, p_{l}\right) \\
R_{u_{l} \pm c_{l}}=\left(\alpha_{v}, u_{v}, p_{v}, s_{v}, s_{l}, u_{l} \pm f_{l}\right)
\end{array}\right.
$$

with $\partial f_{\varphi} / \partial p_{\varphi}=-1 / \rho_{\varphi} c_{\varphi}$

In order to compute approximate solutions of system (14), we use the same non-conservative version of the Rusanov scheme recalled before. Thus, we define :

$$
h_{i}\left(\left(\hat{\alpha}_{l}\right)_{i}^{n}-\left(\alpha_{l}\right)_{i}^{n}\right)+\Delta t\left(g_{i+1 / 2}^{\text {Rusanov }}\left(W_{i}^{n}, W_{i+1}^{n}\right)-g_{i-1 / 2}^{\text {Rusanov }}\left(W_{i-1}^{n}, W_{i}^{n}\right)\right)+\Delta t \mathcal{Q}_{i}^{n}=0
$$

where:

$$
g_{i+1 / 2}^{\text {Rusanov }}\left(W_{i}^{n}, W_{i+1}^{n}\right)=-\tilde{r}_{i+1 / 2}\left(\left(\alpha_{l}\right)_{i+1}^{n}-\left(\alpha_{l}\right)_{i}^{n}\right) / 2
$$

and:

$$
\mathcal{Q}_{i}^{n}=\left(U_{v}\right)_{i}^{n}\left(\left(\bar{\alpha}_{l}\right)_{i+1 / 2}^{n}-\left(\bar{\alpha}_{l}\right)_{i-1 / 2}^{n}\right)
$$

We also keep partial masses and velocities frozen within the step:

$$
\begin{aligned}
& \left(\hat{m}_{k}\right)_{i}=\left(m_{k}\right)_{i}^{n} \\
& \left(\hat{U}_{k}\right)_{i}=\left(U_{k}\right)_{i}^{n}
\end{aligned}
$$

and set :

$$
\begin{aligned}
& \left(\hat{\alpha}_{l} \hat{E}_{l}+\hat{\alpha}_{v} \hat{E}_{v}\right)_{i}=\left(\alpha_{l} E_{l}+\alpha_{v} E_{v}\right)_{i}^{n} \\
& \left(\hat{s}_{l}\right)_{i}=\left(s_{l}\right)_{i}^{n}
\end{aligned}
$$

This substep provides some estimation $\hat{W}_{j}$ within each cell $j$ that is used to initialize the second substep corresponding with (15). We also use the generic Rusanov formulation detailed above to compute approximations of solutions of the second step (15). Positive values of statistical void 
fractions $\alpha_{l, v}$ and partial masses $m_{l, v}$ are guaranteed by the scheme if the time step is constrained by (13). This fractional step method is also meaningful in order to compute approximate solutions of the general system introduced in [16] .

\section{NumERICAL RESUlts}

\subsection{Definition of pure statistical fraction waves and equilibrium solutions}

(1) Some among the following test cases require to define initial conditions (IC) that generate pure statistical fraction waves. These IC are obtained as follows. We assume some given

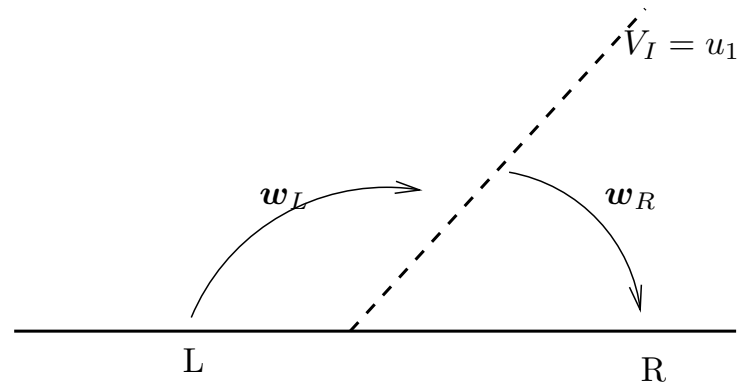

FiguRE 1. Wave Structure of Riemann problem

left state $\boldsymbol{w}_{L}$, and select two values $\left.\left(\alpha_{l}\right)_{R} \in\right] 0,1\left[\right.$ and $\left(\rho_{v}\right)_{R}>0$. Next we enforce the equilibrium of the Riemann invariants of the $1-2$-wave (see property 1 , equation (6)):

$$
I_{1,2}^{k}\left(\boldsymbol{w}_{L}\right)=I_{1,2}^{k}\left(\boldsymbol{w}_{R}\right)=R_{k},
$$

for $k=1$ to 5 . Using these five relations, we get at once that:

$$
\left(u_{v}\right)_{R}=\left(u_{v}\right)_{L}
$$

Next we define the two main unknowns $X=\left(\rho_{l}\right)_{R}$ and $Y=\left(p_{l}\right)_{R}$. Straightforward eliminations enable to establish that the pair $(X, Y)$ should be solution of a system of two non-linear equations:

$$
\begin{aligned}
& s_{l}(X, Y)=R_{2} \\
& e_{l}(X, Y)+\frac{Y}{X}+\frac{R_{3}^{2}}{2\left(\alpha_{l}\right)_{R}^{2} X^{2}}=R_{5}
\end{aligned}
$$

Using (3), it appears that $\partial_{P_{\phi}}\left(s_{\phi}\right) \partial_{\rho_{\phi}}\left(s_{\phi}\right)<0$; hence we may get $Y=\Phi(X)$ solution of the first equation in system (25). Moreover, we know that $\Phi^{\prime}(X)>0$, whatever the EOS of the liquid phase is. Pluging the value $Y=\Phi(X)$ yields:

$$
f(X) \stackrel{\text { def }}{=} e_{l}(X, \Phi(X))+\frac{\Phi(X)}{X}+\frac{A^{2}}{X^{2}}-R_{5}=0
$$


where we note $2 A^{2}=R_{3}^{2} /\left(\alpha_{l}\right)_{R}^{2}$. For non-zero values of $R_{3}$, we define $X_{0}$ solution of $X^{2} \Phi^{\prime}(X)=A^{2}$. The function $f(X)$ is decreasing in the interval ]0, $X_{0}$ ] and then increasing for $X \in\left[X_{0},+\infty[\right.$. Thus system (26) admits two solutions if :

$$
f\left(X_{0}\right)<0
$$

one solution if $f\left(X_{0}\right)=0$, and no solution when $f\left(X_{0}\right)>0$.

Once this pair $(X, Y)$ has been computed, we deduce the remaining two unknowns:

$$
\left(u_{l}\right)_{R}=\left(u_{v}\right)_{L}+\frac{R_{3}}{\left(\alpha_{l}\right)_{R} X}
$$

and:

$$
\left(p_{v}\right)_{R}=\left(R_{4}-\left(\alpha_{l}\right)_{R} Y-\frac{\left(R_{3}\right)^{2}}{\left(\alpha_{l}\right)_{R} X}\right) /\left(1-\left(\alpha_{l}\right)_{R}\right)
$$

In practice, for a stiffened gas EOS in the liquid phase :

$$
e_{l}\left(\rho_{l}, p_{l}\right)=\frac{p_{l}+\gamma_{l}\left(p_{l}\right)_{\infty}}{\rho_{l}\left(\gamma_{l}-1\right)}
$$

with $\gamma_{l}>1$, we may check that $X_{0}$ is unique and also:

$$
X_{0}=\left(\frac{2 A^{2}}{\gamma_{l} R_{2}}\right)^{\left(\frac{1}{\gamma_{l}+1}\right)}
$$

Hence in that case, the equation (26) admits two distinct solutions $X_{1}, X_{2}$ if $f\left(X_{0}\right)<0$.

(2) We also define equilibrium solutions as follows. In that case, initial conditions are chosen such that a pressure/velocity/temperature equilibrium is reached, thus:

$$
\begin{gathered}
p=\left(p_{v}\right)_{L}=\left(p_{l}\right)_{L}=\left(p_{v}\right)_{R}=\left(p_{l}\right)_{R} \\
u=\left(u_{v}\right)_{L}=\left(u_{l}\right)_{L}=\left(u_{v}\right)_{R}=\left(u_{l}\right)_{R} \\
T=\left(T_{v}\right)_{L}=\left(T_{l}\right)_{L}=\left(T_{v}\right)_{R}=\left(T_{l}\right)_{R}
\end{gathered}
$$

The next subsection is devoted to the computation of a classical test case taken from the literature.

\subsection{A classical test case}

This first test case which is taken from [22] corresponds to a one-dimensional Riemann problem. The initial condition (Region L/Region $\mathrm{R}$ ) and the intermediate states 1, 0,2 are presented in table (1). A perfect gas EOS is used for the vapour phase, whereas a stiffened gas EOS is considered for the liquid phase.

Figure 2 shows convergence curves for first and second-order schemes SR1, SR1-ORDER2 on the left, and compares results obtained with scheme SR1 and the fractional step method PFRAC3 


\begin{tabular}{|c|c|c|c|c|c|}
\hline & Region L & Region 1 & Region 0 & Region 2 & Region R \\
\hline$\alpha_{v}$ & 0.3 & 0.3 & 0.8 & 0.8 & 0.8 \\
\hline$\rho_{v}$ & 1.0 & 0.4684 & 0.5030 & 5.9991 & 1.0 \\
\hline$u_{v}$ & -19.5975 & 6.7332 & -1.7541 & -1.7541 & -19.5975 \\
\hline$p_{v}$ & 1000.0 & 345.8279 & 382.0858 & 382.0858 & 0.01 \\
\hline$\rho_{l}$ & 1.0 & 0.7687 & 1.6087 & 1.6087 & 1.0 \\
\hline$u_{l}$ & -19.5975 & -6.3085 & -6.3085 & -6.3085 & -19.5975 \\
\hline$p_{l}$ & 1000.0 & 399.5878 & 466.7257 & 466.7257 & 0.01 \\
\hline
\end{tabular}

TABLE 1. Initial conditions and intermediate states for test case 1 proposed in [22]
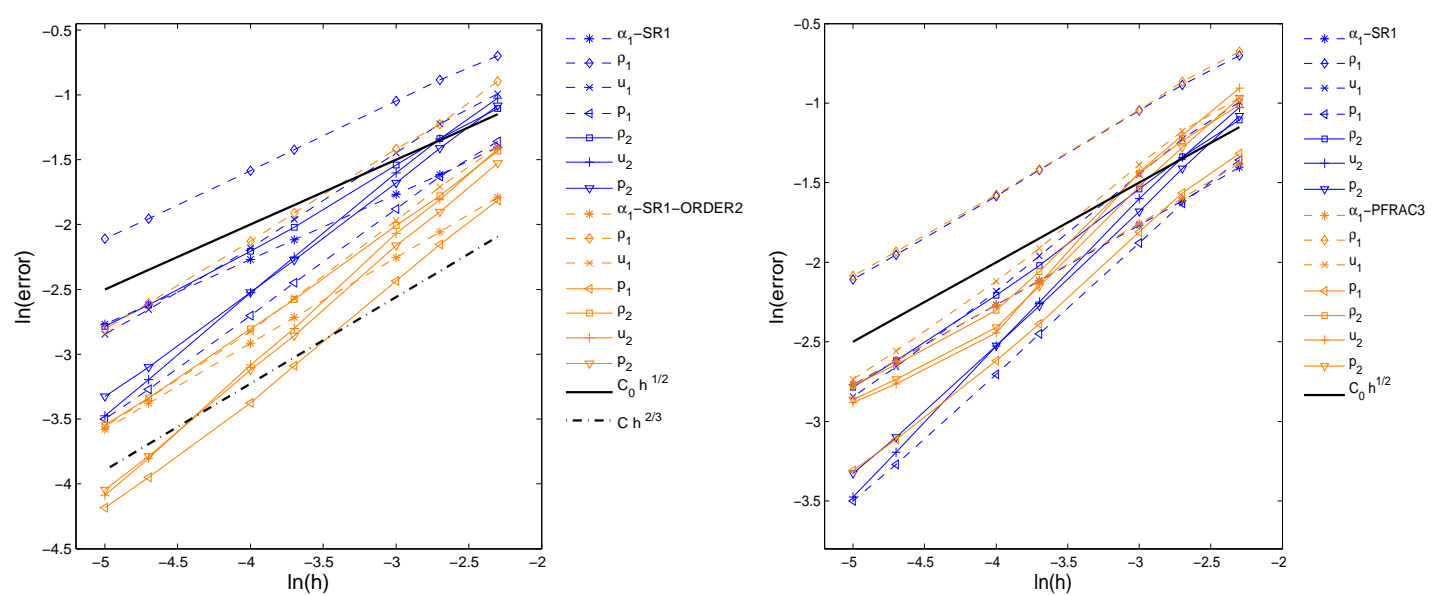

Figure 2. $L^{1}$ norm of the error for $\alpha_{v}, \rho_{\varphi}, u_{\varphi}, p_{\varphi}, \varphi=l, v$, for test case 1 taken from [22], using first and second-order schemes SR1, SR1-ORDER2. PFRAC3. $C F L=0.49, t=0.007$.

on the right. The $L^{1}$ norm of the error has been plotted for all state variables using a logarithmic scale. The slope of the reference black line is $\frac{1}{2}$. Numerical results were obtained at time $t=0.07$ setting $C F L=\frac{1}{2}$. The number of cells in meshes grows from 100 to $10^{5}$. Results that were obtained on coarse meshes ( $200 \& 500$ or $500 \& 5000$ cells) and the exact solution are presented in Figures 3,4 .

Actually, we retrieve the expected rates of convergence $\frac{1}{2}, \frac{2}{3}$ for the first/second order Rusanov schemes. Though the Baer-Nunziato model has no conservative form, we emphasize that it makes sense computing shock waves since the non-conservative products are only active in the linearly degenerate field associated with the 1,2 wave. We note that the three schemes capture the shock wave successfully, and that no oscillations may be observed around shock waves and contact discontinuities. The accuracy of the first-order Rusanov scheme is slightly better than the one of the 
fractional step method on a given mesh, and of course a much better accuracy is gained with the second-order scheme .

\subsection{Pure void fraction waves}

In this subsection, we focus on the behaviour of schemes when computing the void fraction wave.

\subsubsection{Test case 2}

The second test case GPGP1_A3 involves a large initial discontinuity of the statistical phase fraction $\alpha_{v}$ from 0.05 to 0.95 . Two Perfect Gas EOS are used for vapour and liquid phases. The initial condition and the EOS parameters are detailed in table (2). Since the exact solution contains a sole wave associated with $U_{v}$, a unique discontinuity traveling with the velocity $U_{v}$ is expected in the numerical solution between the left and the right states.

\begin{tabular}{|c|c|c|c|}
\hline \multirow{5}{*}{ GPGP1_A3 } & \multicolumn{3}{|c|}{ Exact solution } \\
\cline { 2 - 4 } & & $\boldsymbol{w}_{L}$ & $\boldsymbol{w}_{R}$ \\
\cline { 2 - 4 } & $\alpha_{v}$ & 0.05 & 0.95 \\
\cline { 2 - 4 } & $\rho_{v}$ & 0.1 & 0.1 \\
\cline { 2 - 4 } & $u_{v}$ & 15 & 15 \\
\cline { 2 - 4 } & $p_{v}$ & $1 \times 10^{4}$ & 95044.7776983064 \\
\cline { 2 - 4 } & $\rho_{l}$ & 1 & 0.956131036361501 \\
\cline { 2 - 4 } & $u_{l}$ & 10 & -84.3587660970787 \\
\cline { 2 - 4 } & $p_{l}$ & $1 \times 10^{5}$ & 95185.1409529552 \\
\hline
\end{tabular}

TABlE 2. Second test case GPGP1_A3. Parameters of EOS for vapour and liquid phases: $\gamma_{v}=1.4, \gamma_{l}=1.1$

Computations were run with the first/second order schemes setting $C F L=0.49$ and were stopped at time $t=0.25$. Regular meshes containing from 200 up to $10^{5}$ cells have been used. The expected rates of convergence $\frac{1}{2}$ (respectively $\frac{2}{3}$ ) were again obtained for the first-order scheme

(respectively second-order scheme), see Figure 5. Nonetheless, we observe that the accuracy of the second-order scheme is not much different from the one of the first-order scheme. Actually, numerical results in Figure 6 show some -stable- oscillations around the void-fraction wave with the second-order scheme. This clearly means that the reconstruction is not fully compatible with the Riemann invariants of the $1-2$ wave.

\subsubsection{Third and fourth test cases GPSG1_A3 and GPSG1EQ_A3}

These two test cases GPSG1_A3 involve a pure void fraction wave with a large initial jump of $\alpha_{v}$ from 0.05 to 0.95 . A stiffened gas EOS is used for the liquid, and a perfect gas EOS for the vapour phase. This test case GPSG1_A3 is indeed much more difficult than GPGP1_A3. When applying the first-order Rusanov scheme with $C F L=0.5$, the calculation becomes rapidly unstable, and a restriction on the time step such that $C F L \leq 0.15$ is compulsory in order to reach convergence. We note a huge difference of pressures between the two phases on the right side of the wave (see table 3), and even more that the pressure of the liquid phase becomes negative. Results obtained with the fractional step method are displayed in Figure 9. The fourth test case GPSG1EQ_A3 is even simpler, as shown in table (3), but again approximations obtained on coarse meshes are very 
poor (see Figure 10). The fractional step method performs much better on these two test cases, and we retrieve on Figures 7 and 8 correct rates of convergence for both test cases GPSG1_A3 and GPSG1EQ_A3 at $C F L=0.5$.

\begin{tabular}{|c|c|c|c|}
\hline \multirow{5}{*}{ GPSG1_A3 } & \multicolumn{3}{|c|}{ Exact solution } \\
\cline { 2 - 4 } & & $\boldsymbol{w}_{L}$ & $\boldsymbol{w}_{R}$ \\
\cline { 2 - 4 } & $\alpha_{v}$ & 0.05 & 0.95 \\
\cline { 2 - 4 } & $\rho_{v}$ & 10 & 12 \\
\cline { 2 - 4 } & $u_{v}$ & 15 & 15 \\
\cline { 2 - 4 } & $p_{v}$ & $1 \times 10^{6}$ & 786639.618489141 \\
\cline { 2 - 4 } & $\rho_{l}$ & 1000 & 998.288925495528 \\
\cline { 2 - 4 } & $u_{l}$ & 10 & -80.1628306933727 \\
\cline { 2 - 4 } & $p_{l}$ & $1 \times 10^{6}$ & -3511621.66716409 \\
\hline
\end{tabular}

\begin{tabular}{|c|c|c|c|}
\hline \multirow{4}{*}{ GPSG1EQ_A3 } & \multicolumn{3}{|c|}{ Exact solution } \\
\cline { 2 - 4 } & & $\boldsymbol{w}_{L}$ & $\boldsymbol{w}_{R}$ \\
\cline { 2 - 4 } & $\alpha_{v}$ & 0.05 & 0.95 \\
\cline { 2 - 4 } & $\rho_{v}$ & 5.4766 & 5.4766 \\
\cline { 2 - 4 } & $u_{v}$ & 10 & 10 \\
\cline { 2 - 4 } & $p_{v}$ & $1 \times 10^{6}$ & $1 \times 10^{6}$ \\
\cline { 2 - 4 } & $\rho_{l}$ & 1000 & 1000 \\
\cline { 2 - 4 } & $u_{l}$ & 10 & 10 \\
\cline { 2 - 4 } & $p_{l}$ & $1 \times 10^{6}$ & $1 \times 10^{6}$ \\
\hline
\end{tabular}

TABLE 3 .

-GPSG1_A3, PG (vapour) + SG (liquid) : $\gamma_{v}=1.4, \gamma_{l}=4.4,\left(p_{l}\right)_{\infty}=6 \times 10^{8}$

-GPSG1EQ_A3, PG (vapour) + SG (liquid) : $\gamma_{v}=1.43, \gamma_{l}=2.35,\left(p_{l}\right)_{\infty}=1 \times 10^{9}$

\section{Conclusion}

We have detailed in this paper the numerical simulation of the homogenous system of the twofluid model when considering water-hammer flows. The simple enough Rusanov scheme and a classical extension to second order have been tested for various EOS. Another stable fractional step method is also studied. The following conclusions are :

- We retrieve expected rates of convergence $1 / 2$ and $2 / 3$ for first-order and second-order finite volume schemes respectively, whatever the EOS are;

- Though schemes do not preserve pure statistical fraction waves on coarse meshes, they converge towards the exact solution when the mesh is refined;

- The fractional step method is actually more stable compared with the classical Rusanov scheme, though less accurate than the latter.

Eventually we emphasize that more accurate schemes are necessary in order to achieve cheap enough and reliable flow simulations. Among recent proposals dedicated to the Baer-Nunziato model, we would like to point out the relaxation scheme introduced in $[8,20]$. In addition, for considering water-hammer flows in pipes, liquid-vapour phase transition has to be taken into account. In order to model such phenomena, source terms linked with mechanical relaxation, heat and mass transfer have to be considered in the governing equations. The numerical approximation of this complete two-fluid model can be obtained with the fractional step method that complies with the overall entropy inequality. Such recent algorithms have been introduced in [17] and [18] in order to tackle source terms.

\section{ACKNOWLEDGEMENTS}

The last author has received financial support from EDF and ANRT through a EDF/CIFRE contract 732/2010. Computational facilities have been provided by EDF. 


\section{REFERENCES}

[1] Abgrall R., Karni S., "Computation of compressible multifluids" J. Comp. Physics., vol. 169, pp. 594-623, 2001.

[2] Ambroso A., Chalons C., Coquel F., Galié T., "Relaxation and numerical approximation of a two-fluid two-pressure diphasic model " Math. Model. and Numer. Anal., vol. 43(6), pp. 1063-1098, 2009.

[3] Ambroso A., Chalons C., Raviart P.A., "A Godunov-type method for the seven-equation model of compressible two-phase flow" Computers and Fluids, vol. 54, pp67-91, 2012.

[4] Baer M.R., Nunziato J.W., "A two-phase mixture theory for the deflagration to detonation transition (DDT) in reactive granular materials", Int. J. Multiphase Flow, vol. 12(6), pp. 861-889, 1986.

[5] Baraille R., Bourdin G., Dubois F., Le Roux A.Y., "Une version à pas fractionnaires du schéma de Godunov pour l'hydrodynamique", Comptes Rendus Académie des Sciences, vol. I-314, pp. 147-152, 1992.

[6] Bdzil J.B., Menikoff R., Son S.F., Kapila A.K., Stewart D.S., "Two-phase modeling of a DDT in granular materials: a critical examination of modeling issues", Phys. of Fluids, vol. 11, pp. 378-402, 1999.

[7] Coquel F., Hérard J.M., Saleh K., "A splitting method for the isentropic Baer-Nunziato two-phase flow model " ESAIM proceedings, vol. 38, pp. 241-256, 2013.

[8] Coquel F., Hérard J.M., Saleh K., Seguin N., " A robust entropy-satisfying Finite volume scheme for the isentropic Baer-Nunziato model ", Math. Model. and Numer. Anal., to appear, 2013.

[9] Coquel F., Hérard J.M., Saleh K., Seguin N., " Two properties of two-velocity two-pressure models for two-phase flows", Communications in Mathematical Sciences, to appear, 2013.

[10] GallouËt T., Helluy P., Hérard J.-M., Nussbaum J., "Hyperbolic relaxation models for granular flows", Math. Model. and Numer. Anal., vol.44(2), pp.371-400, 2010.

[11] GallouËt T., Hérard J.-M., Seguin N., "A hybrid scheme to compute contact discontinuities in onedimensional Euler systems", Math. Model. and Numer. Anal., vol.36(6), pp.1133-1159, 2002.

[12] Gallouët T., Hérard J.-M., Seguin N., "Numerical modelling of two phase flows using the two-fluid twopressure approach", Math. Mod. Meth. in Appl. Sci., vol. 14(5), pp. 663-700, 2004.

[13] Gavrilyuk S., Saurel R., "Mathematical and numerical modelling of two phase compressible flows with inertia", J. Comp. Physics., vol. 175, pp. 326-360, 2002.

[14] Girault L., Hérard J.-M., "A two-fluid hyperbolic model in a porous medium", Math. Model. and Numer. Anal., vol. 44(6), pp. 1319-1348, 2010.

[15] HÉrard J.-M., "A three-phase flow model", Mathematical Computer Modelling, vol. 45, pp. 432-455, 2007.

[16] HÉRARD J.-M., "Une classe de modèles diphasiques bi-fluides avec changement de régime", internal EDF report, H-I81-2010-0486-FR, in French, 2010.

[17] Hérard J.-M., Hurisse O., " A fractional step method to compute a class of compressible gas-liquid flows", Computers and Fluids, vol.55, pp. 57-69, 2012.

[18] Hérard J.-M., Hurisse O., " Computing two-fluid models of compressible water-vapour flows with mass transfer", AIAA paper 2012-2959, 2012.

[19] Karni S., Hernandez-Duenas G., "A hybrid algorithm for the Baer Nunziato model using the Riemann invariants", SIAM J. of Sci. Comput., vol.45, pp.382-403, 2010.

[20] SAleh K., Analyse et simulation numérique par relaxation d'écoulements diphasiques compressibles. Contribution au traitement des phases évanescentes. PhD thesis, Université Pierre et Marie Curie, Paris, France, november 26, 2012.

[21] Schwendeman D.W., Wahle C.W., Kapila A.K., "The Riemann problem and a high-resolution Godunov method for a model of compressible two-phase flow ", J. Comp. Physics., vol. 212, pp. 490-526, 2006.

[22] Tokareva S.A., Toro E.F., "HLLC type Riemann solver for the Baer-Nunziato equations of compressible two-phase flow", J. Comp. Physics., vol. 229, pp. 3573-3604, 2010. 

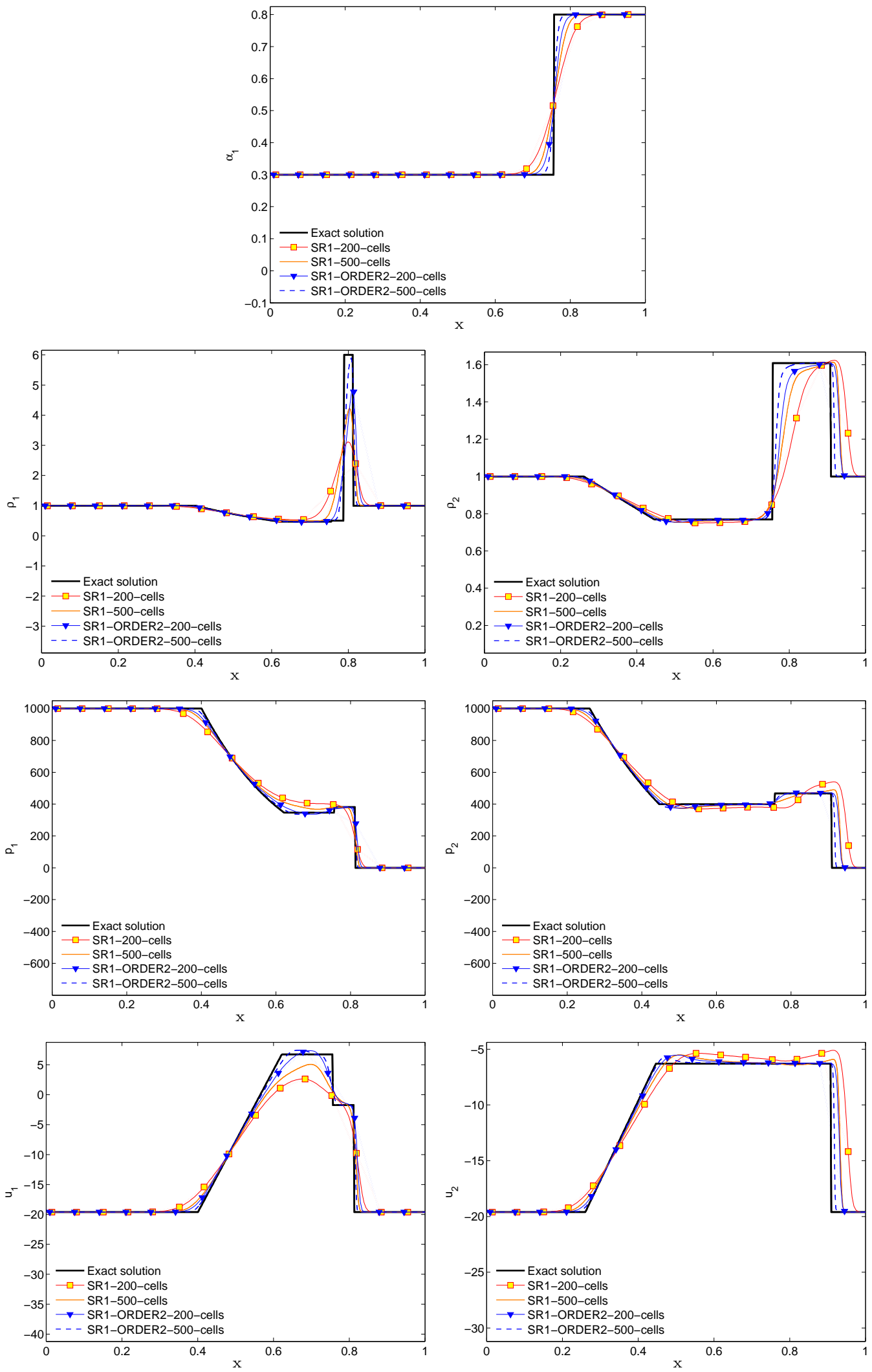

FIgURE 3. Exact solution and numerical approximations of variables $\alpha_{v}, \rho_{\varphi}, u_{\varphi}, p_{\varphi}, \varphi=l, v$ for test case 1, using schemes SR1 and SR1-ORDER2, with 200 and 500 cells. $C F L=0.49, t=0.007$. 

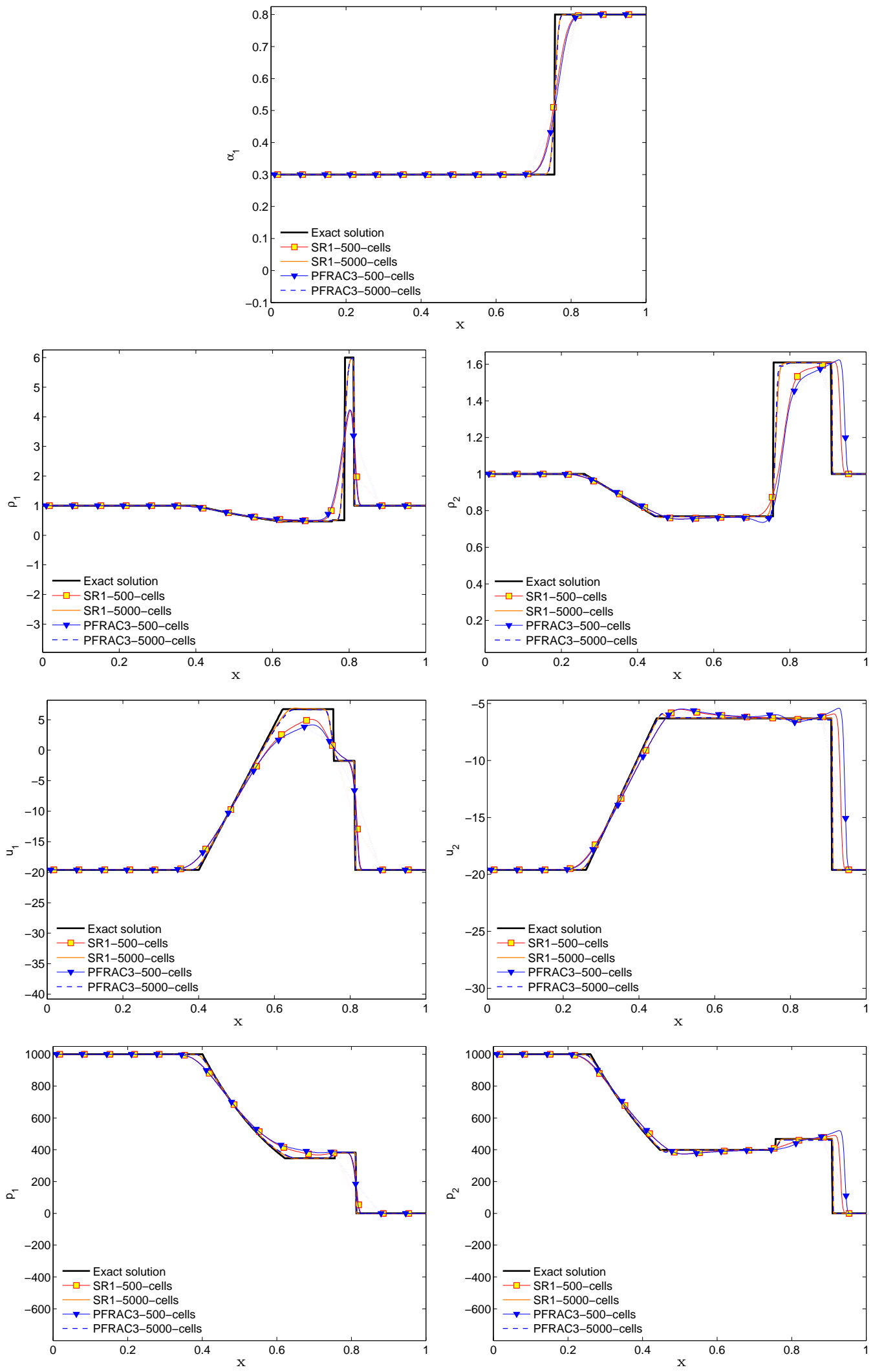

FIgURE 4. Exact solution and numerical approximations of variables $\alpha_{v}, \rho_{\varphi}, u_{\varphi}, p_{\varphi}, \varphi=l, v$ for test case 1 , using schemes SR1 and PFRAC3 with 500 and 5000 cells. $C F L=0.49, t=0.007$. 


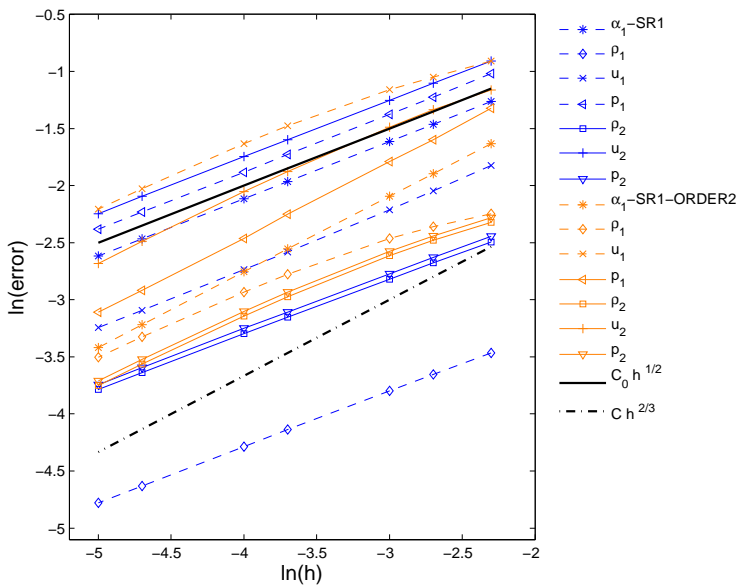

FiguRE 5. $L^{1}$ norm of the error for schemes SR1 and SR1-ORDER2 for the second test case GPGP1_A3. $C F L=0.49, t=0.25$. 

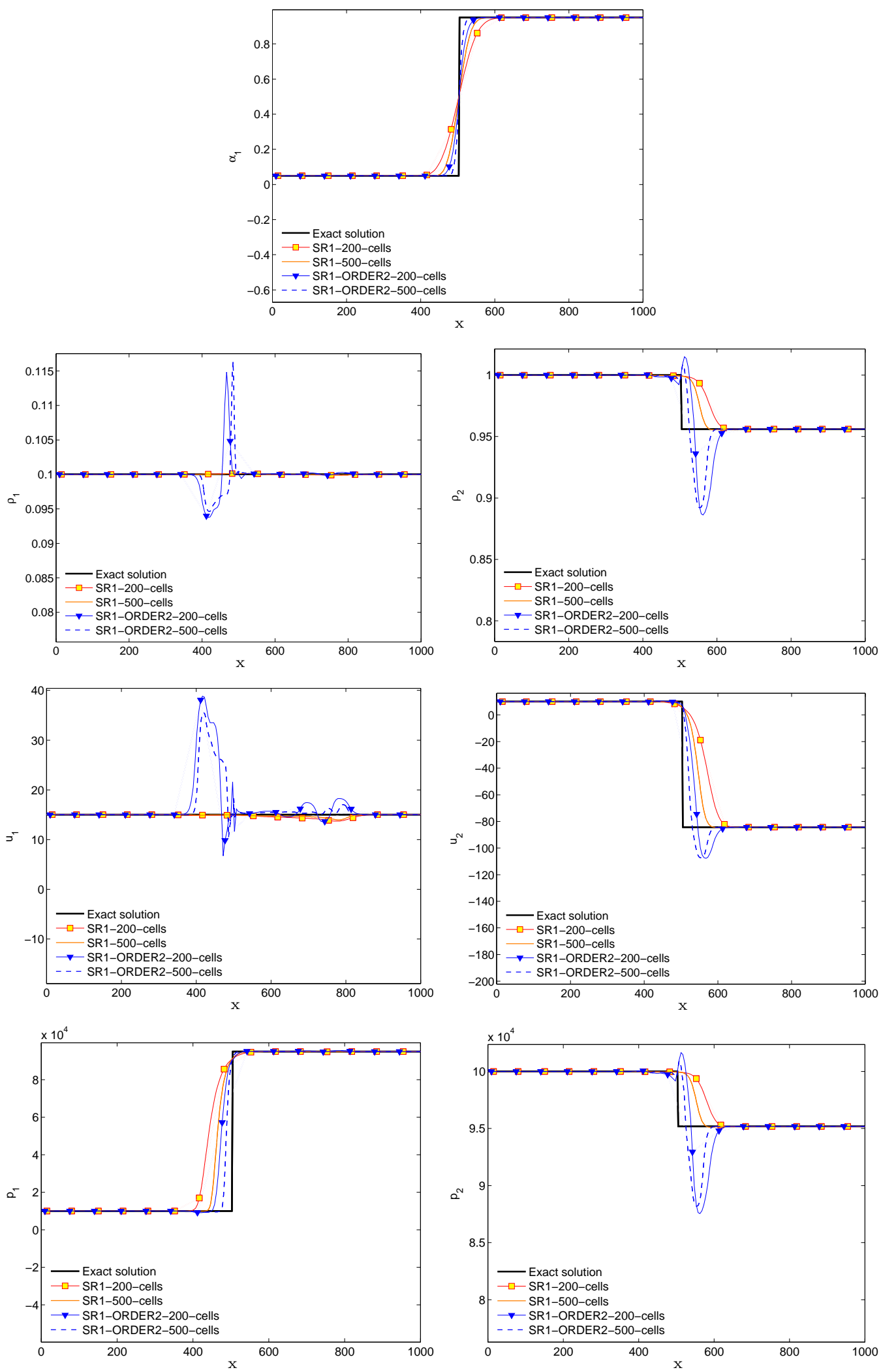

FIgURE 6. Exact solution and numerical approximations for the second test case GPGP1_A3, using schemes SR1, SR1-ORDER2 and meshes containing $200 \&$ 500 elements. $C F L=0.49, t=0.25$. 


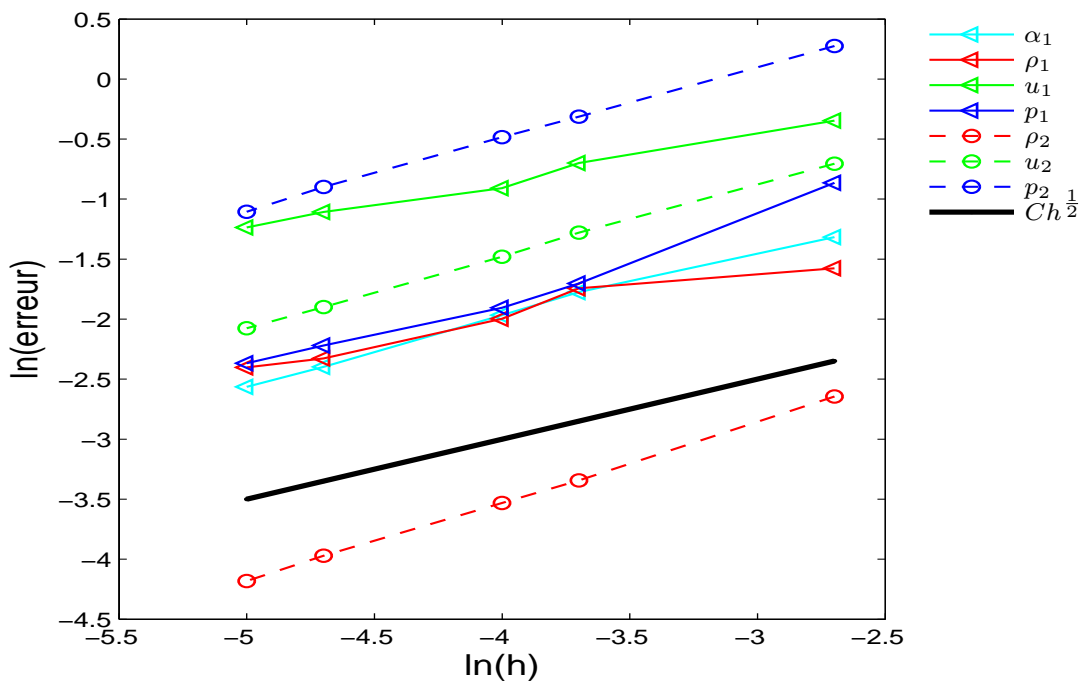

Figure 7. $L^{1}$ norm of the error for scheme PFRAC3 when computing test case GPSG1_A3, with $C F L=0.49, t=0.25$.

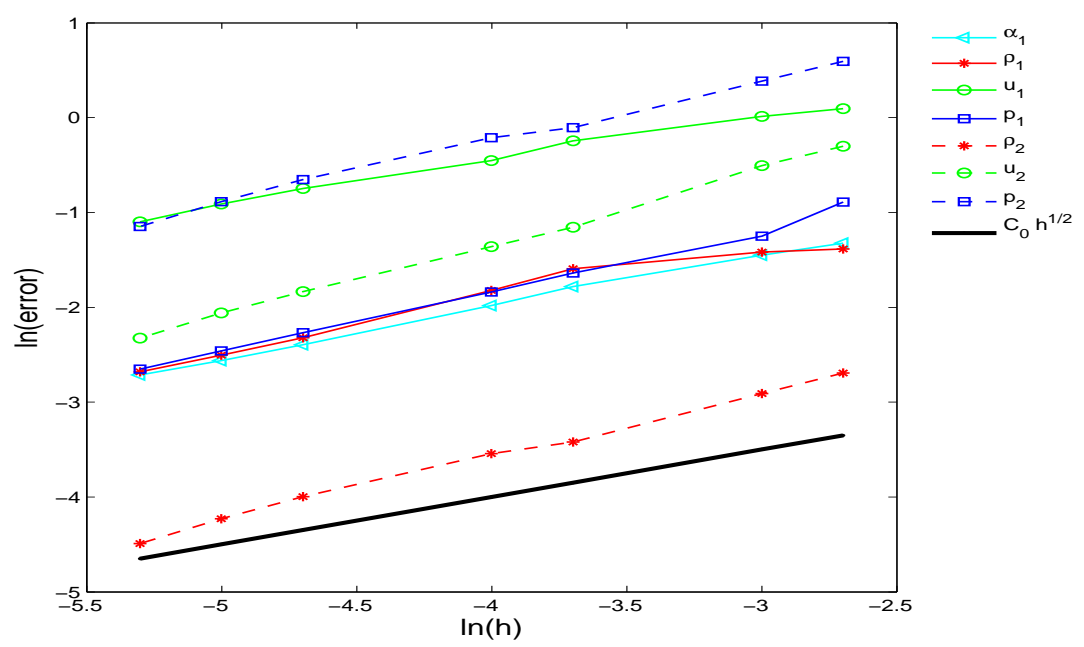

Figure 8. $L^{1}$ norm of the error for scheme PFRAC3 when computing test case GPSG1EQ_A3, with $C F L=0.49, t=0.25$. 

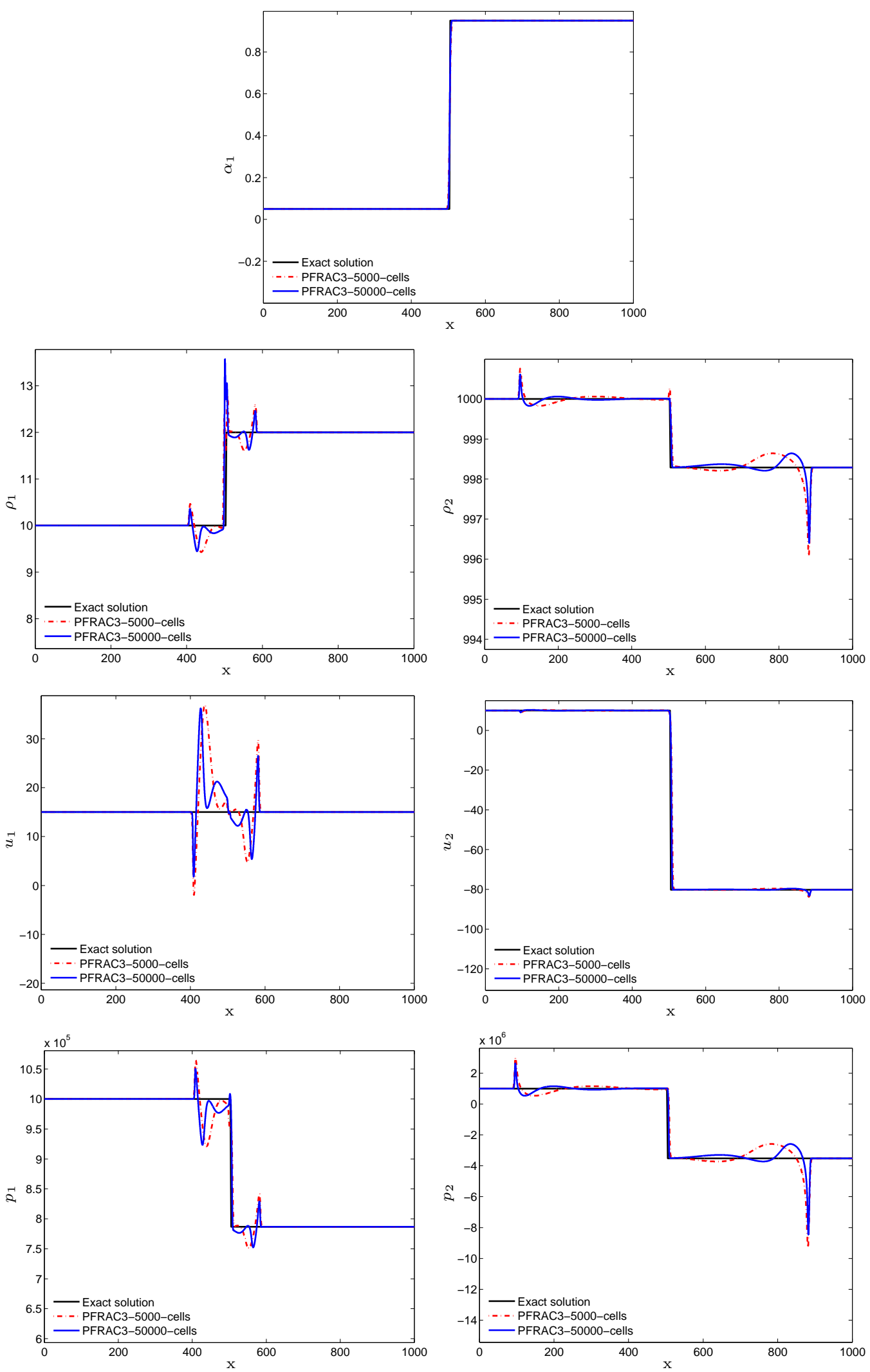

FIGURE 9. Exact solution and numerical approximations for test case GPSG1_A3 with scheme PFRAC3, with meshes containing 5000 \& 50000 cells , CFL = $0.49, t=0.25$. 

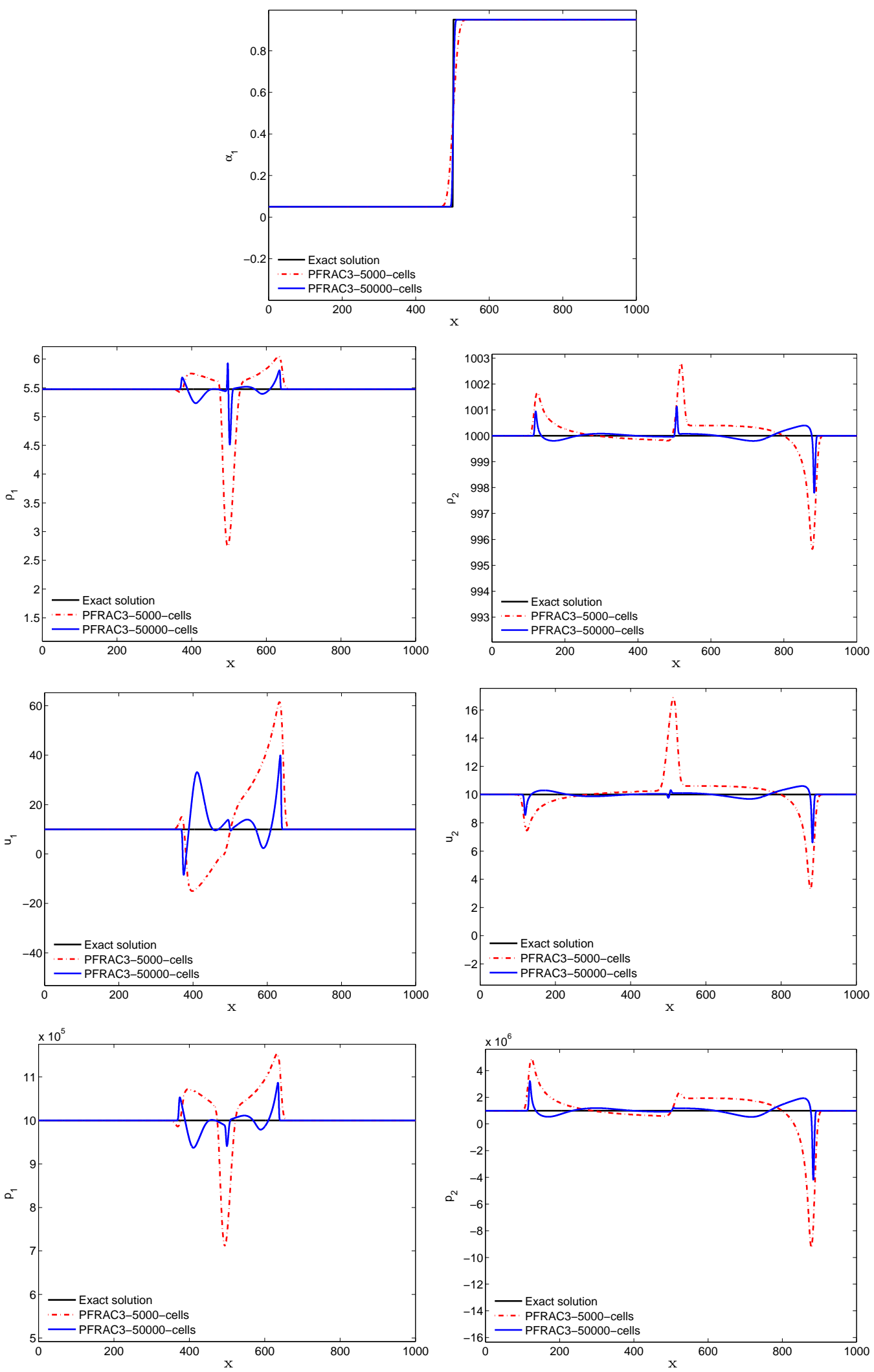

FIGURE 10. Exact solution and numerical approximations for test case GPSG1EQ_A3 with scheme PFRAC3, with meshes containing 5000 \& 50000 cells, $C F L=0.49, t=0.25$. 